\title{
As variações na Comunidade de Odonata (Insecta) em Córregos Podem ser Preditas pelo Paradoxo do Plâncton? Explicando a Riqueza de Espécies Pela Variabilidade Ambiental*
}

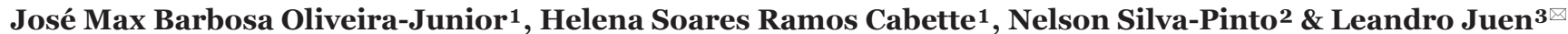

1. Universidade do Estado de Mato Grosso, e-mail: josemaxoliveira@gmail.com, hcabette@uol.com.br 2. Universidade Federal de Goiás, e-mail: nelsonsilvapinto@gmail.com. 3. Universidade Federal do Pará, e-mail: leandrojuen@ufpa.br (Autor para correspondência ${ }^{\bowtie}$ ).

\section{EntomoBrasilis 6 (1): o1-o8 (2013)}

Resumo. A teoria do Paradoxo do Plâncton postula que ambientes que apresentam flutuações temporais periódicas apresentariam alta diversidade de espécie, uma vez que essas flutuações impediriam a ocorrência da exclusão competitiva. Este trabalho teve como objetivo avaliar a variação da comunidade de Odonata adulto na Bacia do Rio Suiá-Missu, testando a hipótese de que locais que apresentam variáveis ambientais com maiores amplitudes de variação possuiriam maiores riquezas de espécies. Foram amostrados 11 corpos d'água, em uma área de transição Cerrado-Floresta Amazônica na região centro-leste do estado de Mato Grosso, Brasil. As variáveis ambientais avaliadas foram integridade ambiental (HII) e amplitude de variação do $\mathrm{pH}$, condutividade, temperatura do ar, temperatura da água, oxigênio dissolvido, amônia, fósforo e Mg+. Foram coletados 2.144 espécimes, distribuídos em oito famílias, 41 gêneros e 78 espécies. Nossa hipótese não foi corroborada, uma vez que a regressão múltipla entre a riqueza estimada de espécie de Anisoptera e Zygoptera e a amplitude de variação dos fatores físico-químicos não foi significativa para nenhuma das variáveis analisadas, assim como para o HII. Nossos resultados sugerem que as variações na comunidade de Odonata em córregos não podem ser explicadas pelo Paradoxo do Plâncton. Acreditamos que este resultado pode ter ocorrido principalmente devido às baixas variações nas condições ambientais analisadas, ação de outros processos locais, como a competição e predação, ou por diferenças ecofisiológicas, resultado da variação de tamanho corporal e da capacidade de termorregulação dos adultos na ordem estudada.

Palavras-Chave: Anisoptera; Heterogeneidade ambiental; Riqueza; Variáveis abióticas; Zygoptera.

\section{Variations in Odonata (Insecta) Community in Streams may be Predicted by the Plankton Paradox? Explaining Species Richness by Environmental Variability}

Abstract. The theory of Plankton Paradox postulates that environments that exhibit regular temporal fluctuations would present a high diversity of species, since such fluctuations would prevent the occurrence of competitive exclusion. This work aimed evaluate variations in adult Odonata community in catchment of River Suiá-Missu, testing the hypothesis that sites with environmental variables with the largest amplitude of variation would present the highest species richness. Were sampled 11 water bodies in an area of transition Cerrado-Amazon Forest in east-central Mato Grosso state, Brazil. Environmental variables evaluated were: environmental integrity (HII) and range of variation of $\mathrm{pH}$, conductivity, air temperature, water temperature, dissolved oxygen, ammonia, phosphorus and $\mathrm{Mg}^{+}$. Were collected 2.144 specimens, distributed in eight families, 41 genera and 78 species. Our hypothesis was not confirmed, since the multiple regression analysis performed between the estimated kind of richness Anisoptera and Zygoptera with range of variation of physical-chemical was not significant for any of the eight variables, as well as for HII. Our results suggest that variations in the community of Odonata in streams cannot be explained by Plankton Paradox. We believe that this result may have occurred due mainly to the low variations in environmental conditions discussed, action of other local processes such as competition and predation or differences ecophysiological result of body size variation and capacity of thermoregulation in the order of the adults studied.

Keywords: Abiotic variables; Anisoptera; Heterogeneity environmental; Richness; Zygoptera.

] eterogeneidade ambiental representa a magnitude da diversidade dehábitats que os organismos podem ocupar ou a diversidade de condições a que um organismo está sujeito em seu hábitat (Chesson 2000; GonZález-Megias et al. 2007). Ambientes heterogêneos permitem a coexistência de um maior número de espécies do que ambientes homogêneos, por disponibilizar maior número de microhábitats e/ou uma gama maior de microclimas (JANKOwsKI et al. 2009). Isto possibilitaria maior segregação no uso dos recursos disponíveis no ambiente e a coexistência de uma quantidade maior de espécies (NESSIMian $e t$ al. 2008), sendo reconhecida como uma das melhores explicações para a variação na diversidade (Houston 1994).

De acordo com Williams et al. (2004) essa coexistência de um maior número de espécies devido a heterogeneidade ambiental funcionaria de maneira similar nos sistemas aquáticos, com a riqueza de espécie aumentando em ambientes mais heterogêneos e mais estáveis, comportando espécies com diferentes exigências ecofisiológicas. Nessas comunidades, os insetos aquáticos se destacam por serem organismos extremamente vulneráreis e, por serem diretamente afetados pelas alterações ambientais, constitui um importante componente da diversidade e integridade de ambientes aquáticos, possibilitando entender à relação entre heterogeneidade e diversidade (RosENBERG \& RESH 1993; Verneaux et al. 2004). Dentre o grupo de insetos aquáticos os indivíduos da ordem Odonata são um dos mais conspícuos, podendo ser afetados tanto por fatores ambientais (JuEN \& DE MARCO 2012), como por estruturais, sendo este último entendido como as estruturas físicas no espaço, promovidas muitas vezes

*Agências de Financiamento: CNPq (proc. no 520268/2005-9); CAPES 
Tp pela presença de galhos, troncos, macrófitas ou outros objetos submersos (Tews et al. 2004; Juen et al. 2007; Woodcock et al. 2007; REIS et al. 2011).

A riqueza de espécies de Odonata ao longo dos rios ou riachos muitas vezes é afetada pela variabilidade natural dos cursos d'água e por condições abióticas proporcionadas pelo gradiente longitudinal dos rios (Clennaghan et al. 1998; FerReira-Peruquetti \& De Marco 2002; Williams et al. 2004) clima regional e altitude. Em uma escala mais local, a distribuição, riqueza e/ ou composição de espécies de Odonata podem ser alteradas devido a presença de vegetação (SAmways \& STeYTLer 1996; Diniz-FilHo et al. 1998), fatores físicos e ou físico-químicos, tais como, temperatura (WARD \& STANFORD 1982), oxigênio dissolvido (Јасов et al. 1984), condutividade (CANnings \& CANnings 1987), hidroperíodo (Wellborn et al. 1996), pH (Courtney \& Clements 1998), correnteza e vazão (CORBET 1999), concentração de poluentes (Couceiro et al. 2007) e outros fatores fortemente ligados a influência antrópica (Oertli 2008; Silva et al. 2010; PINTO et al. 2012).

Adicionalmente, fatores como as perturbações sazonais causadas pelo pulso de inundação também afetam a biota local de Odonata, uma vez que de acordo com sua frequência, intensidade e duração, influenciam na disponibilização de microhábitats (WANTZEN et al. 2008).

Alguns gêneros, como por exemplo Acanthagrion, Cyanallagma, Enallagma, Homeoura, Ischnura e Oxyagrion apresentam hábito escalador e poderiam buscar locais mais protegidos de perturbações ambientais como os pulsos de inundação (Carvalho \& Nessimian 1998). Todos estes fatores parecem ser processos limitantes interferindo tanto na sobrevivência quanto na fecundidade dos adultos (CORBET 1980), podendo limitar até mesmo o tamanho da população local.

Em função disso, Odonata tem sido utilizado tanto em estudos de impacto ambiental quanto em estudos sobre priorização de conservação, no qual diversos estudos têm investigado o aumento ou a diminuição da riqueza e da abundância da ordem, seja por alterações na cobertura vegetal (SAmways \& SteYTLER 1996; REIS et al. 2011; PINTo et al. 2012) ou pelo estado de conservação dos rios (Stewart \& Samways 1998), uma vez que essas alterações levariam a uma homogeneização dos corpos d'água, e seria um dos principais fatores que influenciam as comunidades de organismos em ambientes aquáticos (Ferreira-Peruquetti \& DE
MARCO 2002).

Para explicar a riqueza de espécies em ambientes aquáticos, Hutchinson (1961) em sua teoria do Paradoxo do Plâncton sugeriu a hipótese de que flutuações temporais no ambiente seriam responsáveis pela diversidade de espécies, argumentando que um ambiente natural típico apresenta mudanças em suas condições, muitas delas sazonais como temperatura e umidade, que ocorrem em determinados intervalos de tempo. Tais mudanças podem promover mortalidade indiscriminada dos indivíduos, aliviando os efeitos da dominância competitiva, o que permitira a entrada ou manutenção de espécies pouco competitivas elevando a biodiversidade local (Giacomini 2007).

Com base nos pressupostos de Hutchinson (1961) o objetivo do presente estudo foi avaliar se as variações na comunidade de Odonata podem ser predita pelo modelo do Paradoxo do Plâncton, testando a hipótese de que os locais que apresentam as maiores amplitudes de variação das condições ambientais apresentariam as maiores riquezas de espécies, pois essas variações causariam pequenos distúrbios no ambiente impedindo que algumas espécies conseguissem dominar os recursos.

\section{MATERIAL E MÉTODOS}

Área de estudo. Situada na região centro-leste de Mato Grosso, aproximadamente entre as coordenadas $11^{\circ} 15^{\prime} \mathrm{e} 13^{\circ} 30^{\prime}$ de latitude sul e $51^{\circ} 30^{\prime}$ e $53^{\circ} 15^{\prime}$ de longitude oeste (MAEDA et al. 2008), a Bacia hidrográfica do Rio Suiá-Missu (Figura 1) está localizada em uma área de transição entre os Biomas Cerrado e Floresta Amazônica. A região apresenta clima tropical sazonal com uma estação seca (de maio a outubro) e uma chuvosa (de novembro a abril). O clima da região é predominantemente do subtipo Savana $(A w)$ com microrregiões características do Subtipo Monções $(A m)$ e clima Tropical Chuvoso $(A)$ segundo a classificação de Köppen, com precipitação média anual de cerca de $1.370 \mathrm{~mm}$ e temperatura máxima de $32,7^{\circ} \mathrm{C}$ e mínima de $17,0^{\circ} \mathrm{C}$ (RATTER et al. 1978).

A Bacia do Suiá-Missu abriga trechos ainda preservados de tipos vegetacionais distintos, que segundo RATTER et al. (1978) merecem destaques as fisionomias de cerrado sensu stricto, áreas de Mata Seca e cerrado transicional denominado "cerradão de Hirtella glandulosa" em função da intrigante dominância desta espécie na região (RATTER et al. 1973; CABetTe et al. 2010; Shimano et al. 2010; Nogueira et al. 2011).

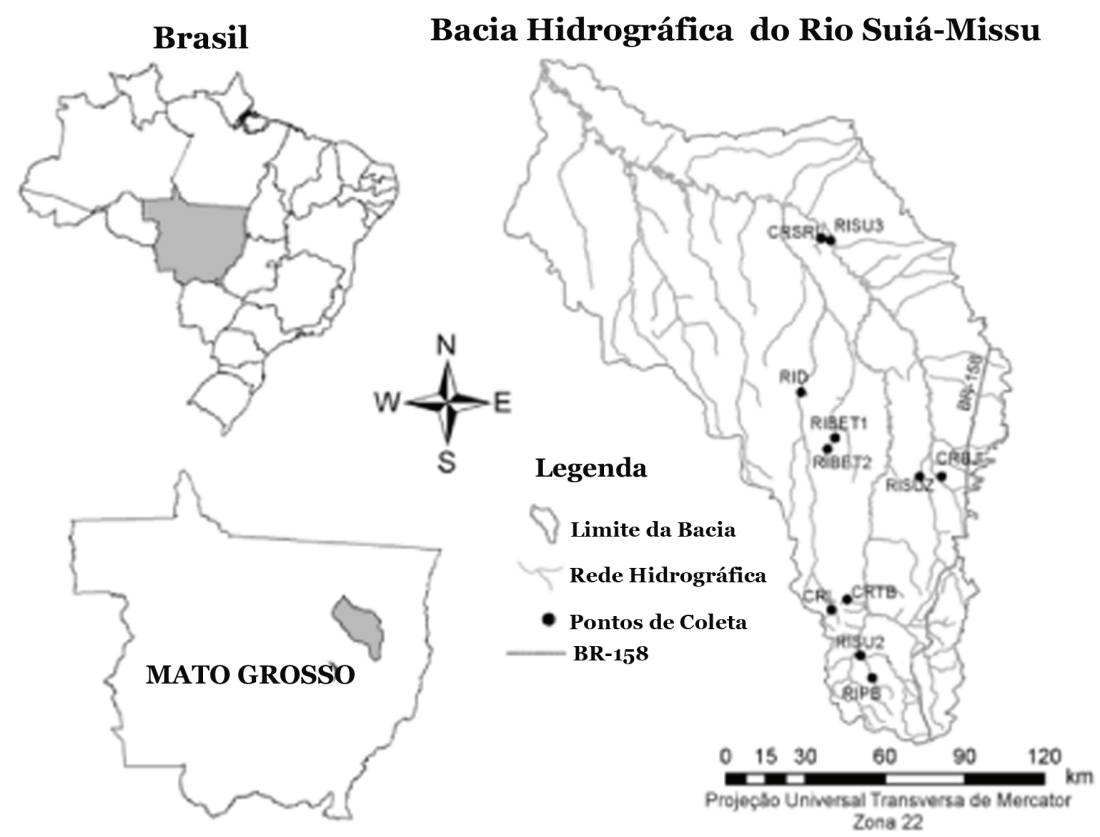

Figura 1. Mapa da área de estudo (Bacia hidrográfica do Rio Suiá-Missu, Mato Grosso, Brasil) mostrando a rede de drenagem e os pontos de coleta: Rio Suiá-Missu local 2 (RISU2), Rio Suiá-Missu local 3 (RISU3), Rio Betis local1 (RIBET1), Rio Betis local 2 (RIBET2), Rio Piabanha (RIPB), Rio Darro (RID), Rio Suiazinho (RISUZ), Córrego Brejão (CRBJ), Córrego Transição-brejo (CRTB), Córrego Sucuri (CRSRI) e Córrego Lúcio (CRL) (adaptado de Pinto et al. 2012). 
Coleta de dados. As coletas foram realizadas em 11 pontos georreferenciados (Figura 1), seis em áreas de cerradão: Córrego Brejão (CRBJ), Córrego Lúcio (CRL), Córrego Transição-brejo (CRTB), Rio Betis 1 (RIBET1), Rio Betis 2 (RIBET2) e Rio Suiazinho (RISUZ), dois em áreas de cerrado senso stricto: Rio Piabanha (RIPB) e Rio Suiá 2 (RISU2) e três em área de mata seca: Córrego Sucuri (CRSRI), Rio Darro (RID) e Rio Suiá-Missu (RISU3). Tais coletas foram realizadas em três períodos do ano: seca (setembro/2007), início das chuvas (dezembro/2007) e vazante (março/2008).

Em cada ponto, as amostragens consistiram na contagem visual do número de indivíduos adultos das espécies de Odonata e na coleta de espécimes para confirmação de identificação com auxílio de rede entomológica (puçá). Para tanto, foram definidos transectos de 100 metros nas margens de cada corpo d'água, os quais foram subdivididos em 20 segmentos de cinco metros, representando pseudorréplicas utilizadas para realização de estimativas da riqueza de espécies.

Este método de varredura (scan) foi definido de acordo com o protocolo de DE MARco (1998), o qual tem sido aplicado em estudos de Libélulas, gerando estimativas pontuais em várias regiões do Brasil (Juen \& De MARCo 2011; Reis et al. 2011; Pinto et al. 2012). O tempo médio de permanência em cada ponto de amostragem foi de uma hora. As coletas foram realizadas em dias ensolarados e com temperatura acima de $19^{\circ} \mathrm{C}$, pois alguns estudos mostram que abaixo dessa temperatura os Odonata diminuem suas atividades de forrageamento (MAY 1976; DE MARCO \& RESENDE 2002). Concomitantemente à coleta das libélulas, foi medida a temperatura do ar à sombra próximo ao corpo d'água.

O acondicionamento e conservação das libélulas coletadas seguem o protocolo descrito em LENCIONI (2006), posteriormente foram depositadas como material testemunho nas coleções Zoobotânica James Alexander Ratter da Universidade do Estado de Mato Grosso, Nova Xavantina-MT e Museu de Zoologia da Universidade Federal do Pará, Belém-PA, Brasil. Para identificação dos espécimes coletados, foram utilizadas chaves sistemáticas (Borror 1945; Belle 1988, 1996; CARvalho \& CALIL 2000; Costa et al. 2002; LeNCIONI 2005, 2006; GARRISON et al. 2006; GARRISON et al. 2010) e comparados com as coleções acima citadas.

Em virtude das diferenças nas exigências ecofisiológicas, biologia e comportamento, nesse estudo as duas subordens Anisoptera e Zygoptera foram avaliadas separadamente, uma vez que espécies da subordem Zygoptera geralmente são indivíduos com maior grau de especificidade, habitam riachos com cobertura vegetal densa, apresentando maior riqueza de espécies em ambientes preservados (CORBET 1999). A mesma teria sua riqueza diminuída em locais alterados, pois os ambientes com grande entrada de luz e consequentemente mais quentes, seriam fatores limitantes para estas espécies, esse efeito não seria homogêneo, mas afetaria grandepartedestesindivíduos, principalmentedevido aotamanho corporal menor (MAY 1976; MAY 1991), consequentemente muitos indivíduos desta subordem (e.g. gêneros como Chalcopteryx, Heteragrion e Protoneura), poderiam superaquecer pelas altas temperaturas ao longo do dia, o que os torna desta forma mais dependentes da temperatura do ar para iniciarem suas atividades (DE MARCo \& RESENDE 2002; MAY 1991). Além disso, variações na estrutura física dos ambientes também são avaliadas devido à importância delas na estruturação da comunidade. Já os Anisoptera por serem maiores, necessitam de áreas com maior incidência solar, sendo assim, a riqueza de espécies desse grupo deve ser maior em ambientes com menor quantidade de vegetação (MAY 1976). Desta forma, devido a tais exigências, as análises por subordens separadamente são mais apropriadas para entender os padrões de riqueza de ambas.

Variáveis ambientais. Em todos os pontos foram aferidas oito variáveis ambientais. Os dados abióticos da água (temperatura da água, $\mathrm{pH}$, condutividade elétrica, oxigênio dissolvido-OD) foram obtidos com auxílio de uma sonda multiparâmetros Horiba ${ }^{\circledR}$. A temperatura do ar foi obtida através de um Termo-Higrômetro digital MT-230 em local sombreado perto do corpo d'água. As concentrações de íons fosfato e amônia foram medidas através de um espectrofotômetro portátil Hach ${ }^{\circledR}$. As concentrações de íons $\mathrm{Mg}^{+}$foram obtidas a partir do método de titulação com EDTA a 0,0002M Método titulométrico do EDTA-Na (NBR 12621/Set, 1992).

As características físicas do ambiente utilizadas apenas para classificar os ambientes quanto ao grau de integridade foram avaliadas usando o procedimento descrito no Índice de Integridade do Hábitat (HII) (Nessimian et al. 2008). Este protocolo é constituído por doze itens que descrevem as condições ambientais avaliando características como: o padrão de uso da terra adjacente à vegetação ribeirinha; largura da mata ciliar e seu estado de preservação; estado da mata ciliar dentro de uma faixa de $10 \mathrm{~m}$; descrição da condição do canal quanto ao tipo de sedimento e presença de dispositivos de retenção; estrutura e desgaste dos barrancos marginais do rio; caracterização do leito do rio quanto ao substrato, vegetação aquática, detritos e disposição das áreas de corredeiras, poções e meandros. Cada item é composto de quatro a seis alternativas ordenadas de forma a representar sistemas cada vez mais íntegros. O valor do índice varia de zero á um, quanto mais próximo de um o valor, mais integro é o sistema (Nessimian et al. 2008).

Análise dos dados. A medida de riqueza foi baseada no estimador não-paramétrico jackknife de primeira ordem, usando o programa Estimates Win 7.5.o (Colwell \& CodDington 1994). Para evitar problemas de multicolinearidade, uma matriz de correlação de Pearson entre todas as variáveis físico-químicas foi calculada, quando a correlação observada entre duas variáveis foi igual ou superior a 0,7 apenas uma dessas foi usada na análise.

Para testar a hipótese de que os locais que apresentam as maiores amplitudes de variação das variáveis ambientais apresentariam as maiores riquezas de espécies utilizamos regressão linear múltipla (ZAR 1999), utilizando como variáveis preditoras a amplitude de variação das variáveis ambientais (obtidas através da comparação de cada variável nas três estações de amostragem) com a riqueza estimada para cada subordem separadamente.

As variáveis ambientais foram transformadas em amplitude por seu valor máximo para equalizar sua contribuição na construção dos eixos, comparando-os nas três estações de amostragem. Os dados físico-químicos foram sumarizados por pontos de coleta pela Análise de Componentes Principais (PCA) de covariância, ou seja, sem padronização por se tratar de amplitude. Para determinar quais componentes principais seriam retidos para análise foi utilizada a aleatoriedade obtida pelo modelo de broken-stick (JACKSON 1993).

Todas as análises, exceto a estimativa de riqueza, foram realizadas pelas rotinas do programa $\mathrm{R}$ (R Development Core TeAm, 2011), utilizando o pacote vegan (OKSANEN et al. 2011).

\section{RESULTADOS}

Descrição da comunidade de Odonata. Foram coletados 2.144 espécimes de Odonata, distribuídos em oito famílias, 41 gêneros e 78 espécies. Das oito famílias, duas pertencem a subordemAnisoptera(Libellulidae eGomphidae)eseisàsubordem Zygoptera (Coenagrionidae, Polythoridae, Protoneuridae, Megapodagrionidae, Calopterygidae, Dicterididae). Dos 41 gêneros, 23 pertencem a subordem Anisoptera, e 18 à subordem Zygoptera (Tabela 1).

A família Protoneuridae (Zygoptera) foi a mais abundante com 716 espécimes. Dos membros desta família, Epipleoneura foi o gênero mais abundante totalizando 447 espécimes, seguindo de Gen.nov. sp.nov Muzon (em descrição) $(n=214)$ e Neoneura 
T $(n=47)$. Dos quatro gêneros mais abundantes, Epipleoneura e Neoneura apresentaram maior abundância em ambientes preservados com grande quantidade de vegetação, por outro lado Gen.Nov. sp.nov apresentou maior frequência em ambientes com pouca vegetação. Megapodagrionidae foi a família de Zygoptera com menos representatividade, sendo esta representada apenas por dois indivíduos de Heteragrion icterops Selys, dos quais um coletado em ambiente com pouca vegetação e outro em ambiente como presença de vegetação ripária (Tabela 1).

Dentro da subordem Anisoptera, Libellulidae foi a família com maior abundância com 482 espécimes coletados, sendo que Eryhtrodiplax $(\mathrm{n}=220)$, Diastatops $(\mathrm{n}=77)$ e Perithemis $(\mathrm{n}=$ 58) foram respectivamente os gêneros mais representativos dessa família. Gomphidae foi a família de Anisoptera com menor abundância, apresentando seis espécimes, sendo que os gêneros Agriogomphus, Phyllocycla e Progomphus apresentaram respectivamente dois espécimes cada (Tabela 1). Todos os indivíduos de Anisoptera foram mais abundantes em ambientes degradados, consequentemente ambientes sem vegetação ripária e mais quentes.

Tabela 1. Relação das espécies e abundância de Odonata, coletadas na bacia do Rio Suiá-Missu, Mato Grosso, Brasil.

\section{Subordens/Famílias/Espécies}

Total de espécimes coletados

\section{ZYGOPTERA}

\section{Calopterygidae}

Hetaerina curvicauda Garrison

180

Hetaerina rosea Selys

Hetaerina westfalli Rácenis

1

Mnesarete aenea (Selys)

Mnesarete machadoi Garrison

\section{Coenagrionidae}

Acanthagrion cuyabe Calvert

Acanthagrion jessei Leonard

Acanthagrion minutum Leonard

Acanthagrion phallicorne Leonard

Acanthagrion truncatum Selys

Argia croceipennis Selys

Argia lilacina Selys

42

17

1

1

Argia reclusa Selys

Argia tinctipennis Selys

Cyanallagma ferenigrum De Marmels

Helveciagrion obsoletum (Selys)

Ischnura capreolus (Hagen)

Oxyagrion fernandoi Costa

Phoenicagrion kajara Machado

Telebasis carminita Calvert

5

Telebasis coccinea (Selys)

Telebasis racenisi Bick \& Bick

Tigriagrion auratinigrum Calvert

\section{Dicteriadidae}

Heliocharis amazona Selys

\section{Megapodagrionidae}

Heteragrion icterops Selys

Polythoridae

Chalcolpteryx rutilans (Rambur)

Protoneuridae

Epipleoneura sp.

Epipleoneura metallica Rácenis

Epipleoneura westfalli Machado

Epipleoneura williansoni Santos

Epipleoneura venezuelensis Rácenis
Subordens/Famílias/Espécies

Total de espécimes coletados

Neoneura denticulata Williamson $\quad 1$

Neoneura gaida Rácenis 3

Neoneura lucas Machado 19

Neoneura luzmarina De Marmels $\quad 19$

Neoneura sylvatica Hagen in Selys $\quad 5$

Phasmoneura exigua (Selys) $\quad 7$

Phasmoneura janirae Lencioni 1

Gen.nov. sp.nov Muzon $\quad 214$

\section{ANISOPTERA}

\section{Gomphidae}

Agriogomphus sp. 2

Phyllocycla armata Belle

Progomphus sp.

Libellulidae

Anatya guttata (Erichson,)

Argyrothemis argentea Ris

Dasythemis sp.

Diastatops intensa Montgomery

Diastatops obscura (Fabricius)

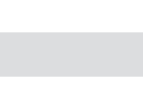

Dythemis sp.

Elasmothemis cannacrioides (Calvert)

9
5
7
1
14

Elga sp.

Erythemis credula (Hagen)

$\begin{array}{ll} & 2 \\ & 6 \\ & 1 \\ \text { (Calvert) } & 42 \\ & 35 \\ & 3 \\ & 2 \\ & 0 \\ & 9\end{array}$

Erythrodiplax amazonica Sjöstedt $\quad 1$

Erythrodiplax basalis (Kirby) $\quad 59$

Erythrodiplax sp. $\quad 8$

Erythrodiplax fusca (Rambur) $\quad 64$

Erythrodiplax juliana Ris $\quad 7$

Erythrodiplax latimaculata Ris $\quad 6$

Erythrodiplax maculosa (Hagen) $\quad 42$

Erythrodiplax ochracea (Burmeister) 18

Erythrodiplax paraguayensis (Förster) 14

Erythrodiplax umbrata (Linnaeus) 1

Fylgia amazonica Kirby $\quad 17$

Idiataphe amazonica (Kirby) 6

Macrothemis imitans Karsch $\quad 1$

Miathyria marcella (Selys in Sagra) $\quad 1$

Miathyria simplex (Rambur) 3

Micrathyria eximia Kirby $\quad 30$

Oligoclada abreviata (Rambur) $\quad 1$

Oligoclada amphinome Ris $\quad 1$

Oligoclada xanthopleura Borror $\quad 13$

Oligoclada walkeri Geijskes $\quad 2$

Orthemis discolor (Burmeister) 4

Perithemis lais (Perty) 54

Perithemis mooma Kirby 4

Planiplax phoenicura Ris $\quad 6$

Tholymis citrina Hagen $\quad 1$

Zenithoptera fasciata (Linnaeus) $\quad 15$

Zenithoptera viola Ris $\quad 3$

Total de indivíduos $\quad 2.144$

Para Zygoptera, RISUZ e CRL foram os pontos com maior riqueza estimada $(21,92 \pm 5,08$ e $19,90 \pm 4,60$ respectivamente) e

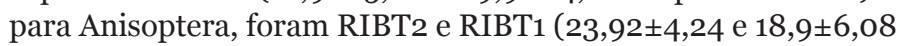
respectivamente) (Tabela 2 ).

Características abióticas dos pontos amostrados. Os ambientes estudados apresentaram águas ácidas $(\mathrm{pH}$ variando 
Tabela 2. Riqueza estimada de Anisoptera e Zygoptera por ponto de coleta na Bacia do Rio Suiá-Missu, Mato Grosso, Brasil.

\begin{tabular}{ccc}
\hline \multirow{2}{*}{ Pontos } & Anisoptera & Zubordens \\
\cline { 2 - 3 } & $11,93 \pm 3,84$ & $13,93 \pm 4,74$ \\
\hline CRBJ & $4,97 \pm 2,76$ & $19,90 \pm 4,60$ \\
CRL & $12,95 \pm 3,34$ & $13,93 \pm 3,84$ \\
CRSRI & $8,97 \pm 2,76$ & $14,95 \pm 3,34$ \\
\hline CRTB & $18,9 \pm 6,08$ & $16,93 \pm 3,84$ \\
\hline RIBT1 & $23,92 \pm 4,24$ & $17,92 \pm 4,24$ \\
\hline RIBT2 & $6,98 \pm 1,96$ & $12,93 \pm 3,84$ \\
\hline RID & $3,97 \pm 2,76$ & $16,92 \pm 4,24$ \\
\hline RIPB & $1,98 \pm 1,96$ & $8,98 \pm 1,96$ \\
\hline RISU2 & $2,98 \pm 1,96$ & $9,95 \pm 3,34$ \\
\hline RISU3 & $8,95 \pm 3,34$ & $21,92 \pm 5,08$ \\
\hline
\end{tabular}

entre 4,11 no CRTB e 6,07 no RISU2), baixa condutividade sendo mais alta no CRBJ, com 1,4 mS/m e menor no CRTB, onde não houve condutividade, alta variação nos valores de fósforo entre 0,08 a 5,82 mg/L, temperatura bastante variável (24,3 no CRL e RISU1 e 29,43 no CRBJ), oxigênio dissolvido (OD) variando entre $1,95 \mathrm{mg} / \mathrm{L}$ no CRTB e 9,81 mg/L no RIPB, mostrando uma considerável variação entre as épocas de coleta, com o período de seca (setembro) fornecendo valores mais baixos na maioria dos locais, com exceção dos RISUZ, RISU1 e RISUZ. Os valores de amônia variaram de -0,02 no CRTB a 0,25 no CRBJ. As concentrações de íons $\mathrm{Mg}^{+}$variaram de 1,81 no CRL a 4,03 no RID.

A associação dos dois eixos da PCA representou $88,99 \%$ da variação ambiental (Eixo 1: 55,19\% e Eixo 2: 33,80\%). As variáveis que mais contribuíram para formação do primeiro eixo foram temperatura do ar e oxigênio dissolvido, relacionadas negativamente com este eixo (Tabela 2, Figura 2). A temperatura da água foi a única variável que mais contribuiu para a formação do segundo eixo, relacionada positivamente ao mesmo (Tabela 3, Figura 2), porém ao realizar um teste-t com os eixos da PCA pode-se observar que não houve uma diferenciação nas variáveis analisadas por ponto de coleta (Eixo 1: $\mathrm{t}=1,689$, g.l. $=9, \mathrm{p}=0,190$; Eixo 2: t=2,007, g.l.=9, $p=0,460)$.

Relação entre a comunidade e as variáveis ambientais. A hipótese de que os locais que apresentam as maiores amplitudes de variação das variáveis ambientais apresentariam as maiores riquezas de espécies não foi corroborada, uma vez que a regressão múltipla realizada entre a riqueza estimada de espécies de Anisoptera e Zygoptera com os fatores físico-químicos não foi significativa para nenhuma das oito variáveis analisadas $\left(\mathrm{r}^{2}=\right.$

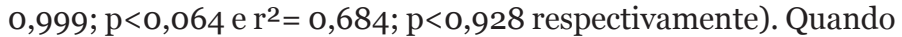
a riqueza estimada de Anisoptera e Zygoptera foi analisada usando somente o HII, as relações observadas também não foram significativas $\left(r^{2}=0,223 ; p=0,142\right.$ e $r^{2}=0,016 ; p=0,712$ respectivamente).

Nossos resultados sugerem que as variações na comunidade de Odonata em córregos não podem ser explicadas pelo Paradoxo do Plâncton, as flutuações temporais (medidas pela amplitude da variação das variáveis ambientais) no ambiente não foram responsáveis por explicar a riqueza estimada nestes ambientes aquáticos.

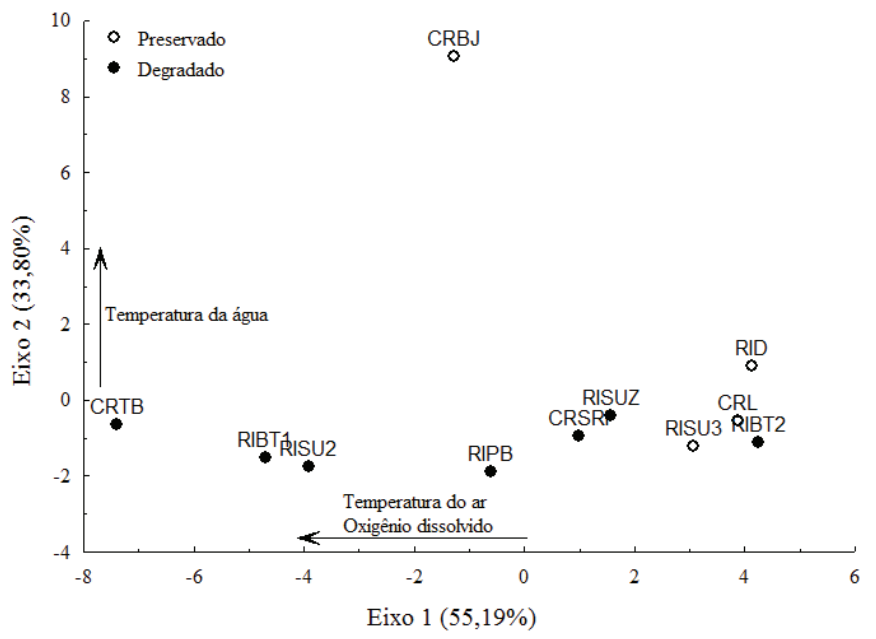

Figura 2. Ordenação das variáveis ambientais por pontos de coleta e tipo de ambiente da Bacia do Rio Suiá-Missu, Mato Grosso, Brasil realizada através de Análise de Componentes Principais (PCA)

Tabela 3. Variáveis ambientais da Bacia do Rio Suiá-Missu, Mato Grosso, Brasil e suas correlações com os Eixos 1 e 2 da Análise de Componentes Principais (PCA) em ambientes preservados e alterados.

\begin{tabular}{|c|c|c|}
\hline \multirow{2}{*}{ Variáveis ambientais } & \multicolumn{2}{|c|}{ Loadings } \\
\hline & Eixo 1 & Eixo 2 \\
\hline $\mathrm{pH}$ & $-0,207$ & $-0,503$ \\
\hline Condutividade & $-0,581$ & 0,285 \\
\hline Temperatura do ar $\left({ }^{\circ} \mathrm{C}\right)$ & $-0,973^{*}$ & $-0,180$ \\
\hline Temperatura da água $\left({ }^{\circ} \mathrm{C}\right)$ & $-0,012$ & $0,991^{*}$ \\
\hline Oxigênio Dissolvido-OD & $-0,834^{*}$ & 0,383 \\
\hline Fósforo & $-0,013$ & 0,233 \\
\hline Amônia & $-0,507$ & 0,494 \\
\hline $\mathrm{Mg}^{+}$ & $-0,271$ & 0,336 \\
\hline Indice de Integridade do Hábitat - HII & 0,207 & $-0,503$ \\
\hline Autovalores & 3,114 & 2,221 \\
\hline Broken-Stick & 2,829 & 1,829 \\
\hline
\end{tabular}

*Considerando ponto de corte segundo Peres-Neto et al. 2003. 


\section{DISCUSSÃO}

As variações ambientais analisadas não exerceram efeito sobre as duas subordens de Odonata, não corroborando assim nossa hipótese de que a variabilidade ambiental apresentaria um forte efeito na riqueza de espécies sendo assim, o modelo proposto por Hutchinson (1961) não foi adequado para explicar a variação de diversidade de espécie observada entre os locais. Acreditamos que isso pode ter ocorrido devido a baixa variação das variáveis mensuradas, bem como as diferenças ecofisiológicas resultado das diferenças de tamanho corporal dos adultos do grupo estudado (CORBET \& MAY 2008).

A comunidade de Odonata apresenta uma grande especificidade de microhabitat estruturado muitas vezes por competição, com os machos passando a maior parte do tempo defendendo os melhores locais para oviposição, sendo esse o handcap que a fêmea utiliza para a escolha do macho (DE MArco et al. 2005). E a exclusão competitiva pode ser evitada pelo grande compartilhamento de recurso entre as espécies (DE MARCo \& RESENDE 2004).

Assim como Hutchinson (1961), Sale (1977), Connel (1978) e Hubbell (1979) também argumentam que as flutuações no meio físico, como mudanças no pH ou na salinidade da água, eventos climáticos severos, como tempestades ou secas prolongadas, agentes físicos como arrebentação de ondas, queda de árvores, entre outros, constituem tipos de distúrbios que evitam essa exclusão devido a diversidade de nichos e impossibilitam as comunidades de se manterem em equilíbrio competitivo na natureza.

Foi observada uma grande representatividade de espécimes da subordem Zygoptera, esta grande representação é comum para ambientes mais sombreados, em virtude de suas habilidades e restrições de termorregulação. Este padrão relaciona-se ao tamanho corporal das espécies desta subordem, em geral pequenos, sendo desta forma por convecção a termorregulação, ocupando locais sombreados como locais de mata (MAY 1991). Já em espécies de tamanho corporal maior, como espécimes da subordem Anisoptera a capacidade de voo em geral é bem maior e consequentemente apresentam uma ampla dispersão e distribuição geográfica (JuEN \& DE MARCo 2011, 2012), nestes espécimes a termorregulação é pela radiação solar (MAY 1991) e desta forma, são mais frequentemente encontrados em áreas abertas.

Pontos que apresentaram maior riqueza estimada foram justamente os pontos com maior alteração ambiental. Outro fator importante a ser considerado é que estes não apresentam cobertura vegetal em seu entorno, o que impulsiona a produção primária, aumentando o número de presas para as formas jovens de Odonata, uma vez que o sucesso desta, em ambientes naturais também está relacionado com a disponibilidade de alimento. Além disso, a presença de grandes quantidades de macrófitas nestes ambientes favorece o aumento da complexidade ambiental o que pode contrabalancear o efeito da diminuição da heterogeneidade ambiental, pois fornece abrigo e proteção às espécies, bem como a formação de microhabitat para as larvas (JuEN et al. 2007), especialmente para aquelas espécies classificadas como escaladores, que necessitam desse tipo de microhabitat para se desenvolverem (NESSIMIAN et al. 2008). Desta forma, o maior número de espécimes encontrados nestes locais, podem ser tanto de áreas alteradas vindas da represa acima como espécies de áreas preservadas.

Este resultado ocorreu principalmente devido às baixas variações nas condições ambientais analisadas, e que a distribuição das espécies pode ser dependente de processos de interação com outras espécies, havendo uma grande divisão de nicho, sendo assim, as oscilações nos parâmetros físico-químicos foram baixas a ponto de não afetarem ou o efeito seria muito pequeno para ser a principal força estruturadora da comunidade. Por outro lado, mudanças nos mecanismos estruturais físicos como a retirada da vegetação marginal dos corpos d'água expõe esses ambientes a elevadas temperaturas e afetam o controle metabólico de organismos ectotérmicos (PusEy \& ARTHINGTON 2003; CASATTI 2010), podendo assim se tornar um efeito drástico e irreversível para a assembleia de Odonata (CoRbet 1999; Fulan \& Henry 2006). Outro fator importante é que até o dado momento a teoria do Paradoxo do Plâncton não havia sido testada com grupos de insetos aquáticos em regiões neotropicais, por isso, o trabalho também se apresenta como uma base para testes que validem o mesmo para estas regiões.

\section{AGRADECIMENTOS}

Ao laboratório de Entomologia da Universidade do Estado de Mato Grosso-UNEMAT pelo apoio institucional e acadêmico e a CAPES pela concessão de bolsa. Aos MSc. Yulie Shimano Feitoza e Denis Silva Nogueira pela contribuição nas análises estatísticas e sugestões para o manuscrito. Aos MSc. Thiago Santos e Guilherme Ferreira de Lima Filho pelo apoio acadêmico e sugestões para o manuscrito. Somos gratos à Lenize Batista Calvão por suas contribuições nas análises dos dados e ao Thiago Mendes pelo auxilio nas coletas. Ao CNPq pelo fomento (proc. $\mathrm{n}^{\mathrm{O}}$ 520268/2005-9). A EMBRAPA, ISA e ONGs relacionadas à Campanha Y Ikatu Xingu, incentivadores deste projeto. Agradecemos também aos dois revisores anônimos por suas sugestões para a melhoria do manuscrito.

\section{REFERÊNCIAS}

Belle, J., 1988. A synopsis of the species of Phyllocycla Calvert with description of four new taxa and a key to the genera of the neotropical Gomphidae (Odonata, Gomphidae). Tijdschrift voor Entomologie, 131: 73-102.

Belle, J., 1996. Higher classification of the South-American Gomphidae (Odonata). Zoologische Mededelingen, 70: 298324.

Borror, D.J., 1945. A key to the New World genera of Libellulidae (Odonata). Annals of Entomological Society of America, 38: 168-194.

Cabette, H.S.R., N.F. Giehl, K. Dias-Silva, L. Juen \& J.D. Batista, 2010. Distribuição de Nepomorpha e Gerromorpha (Insecta: Heteroptera) da Bacia Hidrográfica do Rio Suiá-Miçu, MT: Riqueza relacionada à qualidade do hábitat, p. 113-137. In: Santos, J.E., C. Galbiati \& L.E. Moschini (Eds.). Gestão e Educação Ambiental, Água, Biodiversidade e Cultura. São Carlos, Rima, 426p.

Cannings, R.A. \& S.G. Cannings, 1987. The Odonata of some saline lakes in British Columbia, Canadá: ecological distribuition and zoogeography. Advances in Odonatology, 3: 7-21.

Carvalho, A.L. \& E.R. Calil, 200o. Chaves de identificação para as famílias de Odonata (Insecta) ocorrentes no Brasil - adultos e larvas. Papéis Avulsos de Zoologia do Museu de Zoologia da USP, 41: 423-441.

Carvalho, A.L. \& J.L. Nessimian, 1998. Odonata do Estado do Rio de Janeiro, Brasil: Hábitats e hábitos das larvas, p. 3-28. In: Nessimian, J.L. \& A.L. Carvalho (Eds.). Ecologia de Insetos Aquáticos. Rio de Janeiro, Séries Oecologia Brasiliensis PPGE-UFRJ, 309p.

Casatti, L., 2010. Alterações no Código Florestal Brasileiro: impactos potenciais sobre a ictiofauna. Biota Neotropica, 10: 31-34.

Chesson, P., 2000. Mechanisms of maintenance of species diversity. Annual Review of Ecology and Systematics, 31: 343-366.

Clenaghan, C., P.S. Giller, J. O'Halloran \& R. Hernan, 1998. Stream macroinvertebrate to physic-chemical and biotic factors. Freshwater Biology, 40: 175-193.

Colwell, R.K. \& J.A. Coddington, 1994. Estimating terrestrial biodiversitythroughextrapolation.PhilosophicalTransactions of the Royal Society of London, 345: 101-118.

Connell, J.H., 1978. Diversity in tropical rain forest and coral 
reefs. Science, 199: 1302-1310.

Corbet, P.S., 1980. Biology of Odonata. Annual Review of Entomology, 25: 189-217.

Corbet, P.S., 1999. Dragonflies: Behavior and Ecology of Odonata. Ithaca, NY, Comstock Publishing Associates, 830p.

Corbet, P.S. \& M.L. May, 2008. Fliers and perchers among Odonata: dichotomy or multidimensional continuum? A provisional reappraisal. International Journal of Odonatology, 11: 155-171.

Costa, J.M., A.N. Lourenço \& L.P. Vieira, 2002. Chave de identificação para imagos dos gêneros de Libellulidae citados para o Brasil-Comentários sobre os gêneros (Odonata: Anisoptera). Entomología y Vectores, 9: 477-504.

Couceiro, S.R.M., N. Hamada, S.L.B. Luz, B.R. Forsberg \& T.P. Pimentel, 2007. Deforestation and sewage effects on aquatic macroinvertebrates in urban streams in Manaus, Amazonas, Brazil. Hydrobiologia, 575: 271-284.

Courtney, L.A. \& W.H. Clements, 1998. Effects of acidic pH on benthic macroinvertebrate communities in stream microcosms. Hydrobiologia, 379: 135-145.

De Marco Jr., P., 1998. The Amazonian Campina dragonfly assemblage: patterns in microhabitat use and behaviour in a foraging habitat (Anisoptera). Odonatologica, 27: 239-248.

De Marco Jr., P., A.O. Latini \& D.C. Resende, 2005. Thermoregulatory constraints on behavior: patterns in a neotropical dragonfly assemblage. Neotropical Entomology, 34: 155-162.

De Marco Jr., P. \& D.C. Resende, 2002. Activity patterns and thermoregulation in a tropical dragonfly assemblage. Odonatologica, 31: 129-138.

De Marco Jr., P. \& D.C. Resende, 2004. Cues for Territory Choice in Two Tropical Dragonflies. Neotropical Entomology, 33: 397-401.

Diniz-Filho, J.A.F., C.E.R. Sant'ana \& L.M. Bini, 1998. An eigenvector method for estimating phylogenetic inertia. Evolution, 52: 1247-1262.

Ferreira-Peruquetti, P. \& P. De Marco Jr., 2002. Efeito da alteração ambiental sobre comunidades de Odonata em riachos de Mata Atlântica de Minas Gerais, Brasil. Revista Brasileira de Zoologia, 19: 317-327.

Fulan, J.A. \& R. Henry, 2006. The Odonata (Insecta) assemblage on Eichhornia azurea (Sw.) Kunth (Pontederiaceae) stands in Camargo Lake, a lateral lake on the Paranapanema River (state of São Paulo, Brazil), after an extreme inundation episode. Acta Limnologica Brasiliensia, 18: 99-127.

Garrison, R.W., N. Von Ellenrieder \& J.A. Louton, 2006. Dragonfly Genera of the New Word: an Illustrated and Annotated Key to the Anisoptera. The Johns Hopkins University Press, Baltimore, 368p.

Garrison, R.W., N. Von Ellenrieder \& J.A. Louton, 2010. Damselfly Genera of the New World: an Illustrated and Annotated Key to the Zygoptera. The Johns Hopkins University Press, Baltimore, 490p.

Giacomini, H.C., 2007. Os mecanismos de coexistência de espécies como vistos pela Teoria Ecológica. Oecologia Brasiliensis, 11: 521-543.

González-Megias, A., J.G. Gómes \& F. Sánchez-Piñero, 2007. Diversity-habitat heterogeneity relationship at different spatial and temporal scales. Ecography, 30: 31-41.

Houston, M.A., 1994. The Coexistence of Species on Changing Landscapes. Biological Diversity. Cambridge, Cambridge University Press, 681p.

Hubbell, S.P., 1979. Tree dispersion, abundance, and diversity in a tropical dry forest. Science, 203: 1299-1309.

Hutchinson, G.E., 1961. The paradox of the plankton. The American Naturalist, 95: 137-145.

Jackson, D.A., 1993. Stopping rules in principal components analyses: a comparison of heuristical and statistical approaches. Ecology, 74: 2204-2214.

Jacob, U., H. Walter \& R. Klenke, 1984. Aquactic insects larvae as indicators of limiting minimal content of dissolved oxygen.
Aquatic Insects, 6: 185-190.

Jankowski, J.E., A.L. Ciecka, N.Y. Meyer \& K.N. Rabenold, 2009. Beta diversity along environmental gradients: implications of habitat specialization in tropical montane landscapes. Journal of Animal Ecology, 78: 315-327.

Juen, L., H.S.R. Cabette \& De Marco, P. Jr 2007. Odonate assemblage structure in relation to basin and aquatic habitat structre in Pantanal wetlands. Hydrobiologia, 579:125-134.

Juen, L. \& P. De Marco Jr., 2011. Odonate biodiversity in terrafirme forest streamlets in Central Amazonia: on the relative effects of neutral and niche drivers at small geographical extents. Insect Conservation and Diversity, 4: 1-10.

Juen, L. \& P. De Marco Jr., 2012. Dragonfly endemism in the Brazilian Amazon: competing hypotheses for biogeographical patterns. Biodiversity and Conservation, 21: 3507-3521.

Lencioni, F.A.A., 2005. The Damselflies of Brazil: An Illustrated Guide - The Non Coenagrionidae Families. São Paulo, All Print Editora, 324p.

Lencioni, F.A.A., 2006. The Damselflies of Brazil: An Illustrated Guide - Coenagrionidae. São Paulo, All Print Editora, 419p.

Maeda, E.J., A.R. Formaggio \& Y.E. Shimabukuro, 2008. Análise histórica das transformaçãoes da Floresta Amazônica em áreas agrícolas na Bacia do Rio Suiá-Missu. Sociedade \& Natureza, 20: 5-24.

May, M.L., 1976. Thermoregulation in adaptation to temperature in dragonflies (Odonata: Anisoptera). Ecological Monographs, 46: 1-32.

May, M.L., 1991. Thermal adaptations of dragonflies, revisited. Advances in Odonatology, 5: 71-88.

Nessimian, J.L., E. Venticinque, J. Zuanon, P. De Marco Jr., M. Gordo, L. Fidelis, J.D. Batista \& L. Juen, 2008. Land use, habitat integrity, and aquatic insect assemblages in Central Amazonian streams. Hydrobiologia, 614: 117-131.

Nogueira, D.S., H.S.R. Cabette \& L. Juen, 2011. Estrutura e composição da comunidade de Trichoptera (Insecta) de rios e áreas alagadas da bacia do rio Suiá-Missu, Mato Grosso, Brasil. Iheringia, Série Zoologia, 101: 173-180.

Oertli, B., 2008. The use of dragonflies in the assessment and monitoring of aquatic habitats, p. 79-95. In: Cordoba-Aguilar, A. (Ed.). Model Organisms for Ecological and Evolutionary Research. Oxford, Oxford University Press, 290p.

Oksanen, J., F.G. Blanchet, R. Kindt, P. Legendre, R.B. O’Hara, G.L. Simpson, M.H.H. Stevens \& H. Wagner, 2011. Vegan: community ecology package. Version 1.17-11. Disponível em: http://vegan.r-forge.r-project.org.

Peres-Neto, P.R., D.A. Jackson \& K.M. Somers, 2003. Giving meaningful interpretation to ordination axes: assessing loading significance in principal component analysis. Ecology, 84: $2347-2363$.

Pinto, N.S., L. Juen, H.S.R. Cabette \& P. De Marco Jr., 2012. Fluctuating Asymmetry and Wing Size of Argia tinctipennis Selys (Zygoptera: Coenagrionidae) in Relation to Riparian Forest Preservation Status. Neotropical Entomology, 41: 8390.

Pusey, B.J. \& A.H. Arthington, 2003. Importance of the riparian zone to the conservation and management of freshwater fish: a review. Marine and Freshwater Reserch, 54: 1-16.

Ratter, J.A., G.P. Askew, R.F. Montgomery \& D.R. Gifford, 1978. Observations on Vegetation of Northeastern Mato-Grosso. Forests and Soils of Rio Suiá-Missu area. Proceedings of the Royal Society of London Series B-Biological Sciences, 203: 191-208.

Ratter, J.A., P.N. Richards, G.E. Argente \& D.R.G. Giford, 1973. Observations on the vegetation of northeastern Mato Grosso. Philosophical Transaction of the Royal Society of London Series B - Biological Sciences, 226: 449-492.

R Development Core Team, 2011. R: A language and environment for statistical computing. [2.12.2] Vienna: $\mathrm{R}$ Foundation for Statistical Computing. Disponível em: http://www.r-project.org.

Reis, E.F., N.S. Pinto, F.G. Carvalho \& L. Juen, 2011. Efeito 
da integridade ambiental sobre a Assimetria Flutuante em Erythrodiplax basalis (Libellulidae: Odonata) (Kirby). EntomoBrasilis, 4: 103-107.

Rosenberg, D.M. \& V.H. Resh, 1993. Introduction to freshwater biomonitoring and benthic macroinvertebrates, p. 1-9. In: Rosenberg, D.M. \& V.H. Resh, (Eds.). Freshwater Biomonitoring and Benthic Macroinvertebrates. New York, Chapman and Hall, 504p.

Sale, P.F., 1977. Maintenance of high diversity in coral reef fish communities. The American Naturalist, 111: 337-359.

Samways, M.J. \& N.S. Steytler, 1996. Dragonfly (Odonata) distribution patterns in urban and forest landscapes, and recommendations for riparian management. Biological Conservation, 78: 279-288.

Shimano, Y., H.S.R. Cabette, F.F. Salles \& L. Juen, 2010. Composição e distribuição da fauna de Ephemeroptera (Insecta) em área de transição Cerrado-Amazônia, Brasil. Iheringia, Série Zoologia, 100: 301-308.

Silva, D.P., P. De Marco Jr. \& D.C. Resende, 2010. Adult odonate abundance and community assemblage measures as indicators of stream ecological integrity: A case study. Ecological Indicators, 10: 744-752.

Stewart, D.A.B. \& M.J. Samways, 1998. Conserving dragonfly (Odonata) assemblages relative to river dynamics in an African savanna game reserve. Conservation Biology, 12: 683-692.

Tews, J., U. Brose, V. Grimm, K. Tielbor-Ger, M.C. Wichmann, M. Schwager \& F. Jeltsch, 2004. Animal species diversity driven by habitat heterogeneity/diversity: the importance of keystone structures. Journal of Biogeography, 31: 79-92.

Verneaux, V., J. Verneaux, A. Schmitt, C. Lovy \& J.C. Lambert, 2004. The Lake Biotic Index (LBI): an applied method for assessing the biological quality of lakes using macrobenthos; the Lake Châlain (French Jura) as an example. Annales de Limnologie-International Journal of Limnology, 40: 1-9.

Wantzen, K.M., W.J. Junk \& K.O. Rothhaupt, 2008. An extension of the floodpulse concept (FPC) for lakes. Hydrobiologia, 613: 151-170.

Ward, J.V. \& J.A. Stanford, 1982. Thermal responses in the evolutionary ecology of aquatic insects. Annual Review of Entomology, 27: 97-117.

Wellborn, G.A., D.K. Skelly \& E.E. Werner, 1996. Mechanisms creating community structure across a freshwater habitat gradient. Annual Review of Ecology, Evolution, and Systematics, 27: 337-363.

Williams, P., M. Whitfield, J. Biggs, S. Bray, G. Fox, P. Nicolet \& D. Sear, 2004. Comparative biodiversity of rivers, streams, ditches and ponds in an agricultural landscape in Southern England. Biological Conservation, 115: 329-341.

Woodcock, B.A., S.G. Potts, D.B. Westbury, A.J. Ramsay, M. Lambert, S.J. Harris \& V.K. Brown, 2007. The importance of sward architectural complexity in structuring predatory and phytophagous invertebrate assemblages. Ecological Entomology, 32: 302-311.

Zar, J.H., 1999. Biostatistical Analysis. Prentice-Hall, Englewood Cliffs, N.J. 944p.

\section{Recebido em: 18/o5/2012}

Aceito em: 25/11/2012

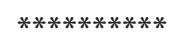

Como citar este artigo:

Oliveira-Junior, J.M.B., H.S.R. Cabette, N. Silva-Pinto \& L. Juen, 2013. As variações na Comunidade de Odonata (Insecta) em Córregos Podem ser Preditas pelo Paradoxo do Plâncton? Explicando a Riqueza de Espécies Pela Variabilidade Ambiental. EntomoBrasilis, 6(1): 01-08.

Acessível em: http://www.periodico.ebras.bio.br/ojs/index.php/ebras/article/view/250. doi:10.12741/ebrasilis.v6i1.250 